\title{
Analysis of the X-Factor and X-Factor stretch during the completion of a golf practice session in low-handicap golfers
}

Graeme G. Sorbie

Yaodong Gu

Julien S. Baker

Ukadike C. Ugbolue

Sorbie, G.G., Gu, Y., Baker, J.S. \& Ugbolue, U.C.

Analysis of the X-Factor and X-Factor stretch during the

completion of a golf practice session in low-handicap

golfers. International Journal of Sports Science and

Coaching.

(C) 2018 the authors

Reprinted by permission of SAGE Publications.

DOI: https://doi.org/10.1177/1747954118791330 
1 Original Research

2 Corresponding Author:

3 Graeme G Sorbie, Biomechanics Laboratory, Division of Sport and Exercise Sciences,

4 Abertay University, Dundee, DD1 1HG, United Kingdom, Phone: +44 (0)1382308015,

$5 \quad$ Email: g.sorbie@uad.ac.uk

\section{Analysis of the $\mathrm{X}$-Factor and X-Factor stretch during the}

\section{7 completion of a golf practice session in low-handicap golfers}

Graeme G. Sorbie, ${ }^{1,2}$ Yaodong Gu, ${ }^{3}$ Julien S. Baker, ${ }^{1,3}$ and Ukadike C. Ugbolue, ${ }^{1,4}$

$8{ }^{1}$ School of Science and Sport, Institute for Clinical Exercise \& Health Science, University of

9 the West of Scotland, Hamilton, ML3 0JB, United Kingdom

$10{ }^{2}$ Division of Sport and Exercise Sciences, Abertay University, Dundee, DD1 1HG, United

11 Kingdom

$12 \quad{ }^{3}$ Faculty of Sports Science, Ningbo University, China

${ }^{4}$ Department of Biomedical Engineering, University of Strathclyde, Glasgow, United

14 Kingdom 


\section{Abstract}

2 The X-Factor and X-Factor stretch have been positively correlated with golf long game

3 performance. The aim of this study was to compare the X-Factor, X-Factor stretch and long

4 game performance variables pre and following a golf practice session. A group of male golfers

$5 \quad(\mathrm{n}=15$, handicap $=3.3 \pm 1.7)$ participated in the laboratory-based-study. Movement and

6 performance variables were collected from five golf swings performed pre and following a golf

7 practice session using a motion capture system and launch monitor respectively. Following the

8 practice session, significant increases were observed in the X-Factor $(p=0.00, d=0.22)$ and

9 the X-Factor stretch $(p=0.02, d=0.25)$. Specifically, the X-Factor increased from $52.82 \pm$ $5.64^{\circ}$ to $54.06 \pm 5.61{ }^{\circ}$ following the practice session. The $\mathrm{X}$-Factor stretch increased from $1.54 \pm 1.05^{\circ}$ to $1.90 \pm 1.41^{\circ}$ following the practice session. Significant differences were displayed in club head velocity $(p=0.00, d=0.35)$, ball velocity $(p=0.01, d=0.21)$ and actual carry distance $(p=0.00, d=0.29)$ following the practice session. These findings suggest that performing multiple golf shots is not detrimental in terms of muscular fatigue in the long game performance. In actual fact, the findings demonstrate that performing 100 golf shots increases the X-Factor, X-Factor stretch patterns and performance variables which, in turn, increases long game performance. These findings can help PGA golf Professionals improve teaching practices and formulation of golf programmes and warm-up sessions.

Key words: Flexibility, ROM, Performance, Movement, Biomechanics 
2 Similar to many other sports, there are several different ways to improve performance in golf;

3 including technique changes and physiological improvements. ${ }^{1}$ The importance of long game

4 performance within the game of golf is widely recognised within the golfing literature. Long

5 game performance takes into consideration golf shots that are performed with a full swing. This

6 has recently intensified due to the rise in specific physical conditioning and warm-up routines

7 at the elite level..$^{2-5}$ These physical conditioning and warm-up studies have mainly focused on

8 improving range of motion (ROM), stability and balance in order to improve movement

9 patterns, including the X-Factor, and in turn aim to increase long game performance..$^{3,4}$

Many of the studies that have focused on the physical conditioning of golfers have investigated key components of long game performance including: club head velocity, ${ }^{2,3,5,6}$ ball velocity, ${ }^{7,8}$ carry distance "distance travelled by the ball in the air" "and kinematic changes during the golf swing. ${ }^{10-13}$ These studies have reported increases in long game performance variables as a result of performing golf specific conditioning and warm-up programmes. ${ }^{1,4-6}$ Specifically, Thompson et al. ${ }^{3}$ reported a significant increase in club head velocity following a functional training intervention. Similarly, Lephart et al. ${ }^{4}$ investigated the effect of an 8 -week golf stretching exercise programme. These authors reported a significant increase in: club head velocity, ball velocity, carry distance, pelvis rotation and X-Factor velocity following the intervention.

The X-Factor and X-Factor stretch have been extensively used within the golf scientific research. ${ }^{4,9,11,14,15}$ The X-Factor describes the rotation of the torso relative to the pelvis at the top of the backswing. ${ }^{16}$ Golfers that are able to exhibit a larger X-Factor are shown to increase club head velocity and ball velocity at impact. ${ }^{4,11}$ Within the studies that reported X-Factor, angles typically range between $30-55^{\circ}$, however, this is dependent on the skill level of the 
1 golfer as previously discussed. ${ }^{4,15}$ The X-Factor stretch has been used to describe the additional

2 rotation that occurs at the start of the downswing. ${ }^{15}$ The X-Factor stretch is caused by the

3 initiation of the pelvic rotation prior to the rotation of the torso. ${ }^{15}$ It has been noted previously

4 that golfers who exhibit this stretch at the beginning of the downswing through dynamic tension

5 of the torso muscles have a greater club head velocity. ${ }^{4}$ Within highly skilled golfers, X-Factor

6 stretch angles typically range between $1-5^{\circ} .9$

7 In order to exhibit an increased X-Factor, golfers must demonstrate a good level of $\mathrm{ROM}^{17}$,

8 which is promoted through flexible muscles and joints. ${ }^{18}$ Flexibility has been found to decrease

9 resistance to swing plane and decrease stretch reflex. ${ }^{18}$ These factors enable an increased ROM

10 in the backswing ${ }^{19}$ and a reduction in injury. ${ }^{20}$ It has been previously reported that performing warm-up techniques before a competition can increase ROM and, therefore, increase the performance of the athlete. ${ }^{21,22}$ In addition to these findings, Joyce ${ }^{8}$ reported that axial rotation flexibility variables of the trunk were associated with club head velocity, which may help facilitate a greater ROM and may create a greater X-Factor. Although previous research has shown that performing warm-up techniques prior to a competition can increase ROM and in turn increase performance, the golf literature is somewhat contrasting. ${ }^{23,24}$ Henry et al. ${ }^{15}$ reported that performing a golf specific dynamic rotational warm-up prior to golf competition had no effect on the X-Factor or X-Factor stretch. In contrast, Gergley ${ }^{23}$ reported a dynamic golf club warm-up significantly increased club head velocity and distance in comparison to a passive static stretching warm-up. These improvements are thought to be a result of increased ROM and a more compliant muscle tendon unit. The contrasting findings within the literature may be a result of the different warm-up routines examined. Although Henry et al. ${ }^{15}$ examined a dynamic rotational warm-up, which has similarities to the golf swing, Gergley ${ }^{23}$ examined a warm-up routine that incorporated golf swings within the process. This may have a greater effect on long game performance due to the greater specificity of the task being performed. ${ }^{23}$ 
1 With the contrasting findings within the golf literature and the limited research on performing

2 multiple golf shots, it is unknown if the X-Factor at the top of the backswing or the X-Factor stretch during the initiation of the downswing changes as a result of performing these golf shots. During golf practice or competition, golfers may perform the golf swing 100 times or

5 more, ${ }^{25}$ which includes golf shots when hitting the ball and practice swings (based on golf 6 handicap). It is possible that multiple golf swings during golf practice or warm-up routine may 7 increase ROM of the torso and pelvis regions at the top of the backswing and, therefore, 8 increase the X-Factor and X-Factor stretch. These swings, however, may also have a negative effect on the separation between these segments by inducing fatigue in the muscles which are highly active during the golf swing, resulting in a reduced ROM, which in turn reduces the XFactor, X-Factor stretch and long game performance variables. ${ }^{26}$

Due to the limited knowledge regarding the effect that multiple golf swings can have on the Xfactor and X-Factor stretch, the purpose of the current study was to assess what effect the golf practice session has on the X-factor, X-Factor stretch and performance variables. The aim of the current study was to describe the X-Factor and X-Factor stretch patterns during the golf swings performed pre and post golf practice session. Additionally, the current study aimed to describe the changes, if any, in club head velocity, ball velocity and actual carry distance from the golf swings performed pre and post golf practice session. A null hypothesis for all variables was adopted due to the conflicting views on the topic area.

Methods

\section{Participants}

Fifteen right-handed male golfers participated in this study (height: $186.0 \pm 5.3 \mathrm{~cm}$, weight: $80.9 \pm 6.9 \mathrm{~kg}$, age: $23.8 \pm 2.9$ years, British Golf Association handicap: $3.3 \pm 1.7$ ). Additional anthropometric measurements (leg length, knee width, ankle width, shoulder offset, elbow 
1 width, wrist width, and hand thickness) were recorded during the initial stages of the testing

2 process in order to run a customised Golf Model. Measurements were recorded to the nearest $\mathrm{mm}$. Participants were required to have no history of lower back pain and/or persistent musculoskeletal disorders. These factors may limit flexibility and golf swing attributes. ${ }^{20}$ Participants were also required to undertake no conditioning or resistance training 48 hours before the testing session. In addition to these specific requirements, participants were required to exhibit a 'modern' golf swing rather than a 'classic swing'. This was assessed by a Professional Golfers' Association (PGA) member. The main differences between the two swing types is that the 'classic' swing exhibits lead heel raise during the backswing, resulting in different pelvic movements, hence the reason why this swing was excluded from the current study. ${ }^{8}$ All participants completed a consent form and physical readiness questionnaire before participating in the current study. The University of the West of Scotland, School of Science and Sport Ethics Committee granted ethical approval for the study to take place (Approval number: 5-3-14-002).

\section{Experimental Procedure}

Prior to data collection, participants performed a standardised golf specific warm-up. Dynamic stretches were performed which targeted specific musculature highly active during the golf swing and was followed by five air swings. Following the warm-up, participants performed five golf shots using the TaylorMade driver ${ }^{8,27,28}$ (TaylorMade, Basingstoke, UK) and a premium golf ball. After these initial five golf shots were completed, participants then completed a typical practice session, hitting 50 golf shots with the driver and 50 golf shots with the TaylorMade 7-iron (TaylorMade, Basingstoke, UK). Prior to the testing all participants were given five minutes to familiarise themselves with the TaylorMade golf clubs. ${ }^{1}$ Utilizing the identical clubs throughout the study removed the effect of the golf club. After the practice session, participants hit five golf shots with the driver again. ${ }^{26}$ Before hitting shots, participants 
1 were informed of what the practice session would entail and advised to take into consideration

2 their average distance when using the 7 -iron and driver. ${ }^{29}$ During the practice session, all golf shots in the session were hit at a rate of one shot every $30 \mathrm{~s}$. During an initial pilot study, golfers stated that this was a comfortable pace to perform the golf shots. In order for all golf shots to

5 be hit safely during the testing session, shots were hit from an artificial golf mat (Longridge,

6 United Kingdom), which was placed in the centre of the laboratory, towards an enclosed golf net (Sports Net Company, United Kingdom). When performing golf shots, participants were advised to aim towards a red target pole, which was situated behind the enclosed golf net.

\section{Data Collection}

During the five golf shots that were performed pre and post the practice session, three dimensional (3-D) data were collected using an 8-camera Vicon Bonita (Oxford Metrics Ltd, United Kingdom) Motion Analysis System operating at $250 \mathrm{~Hz}$ positioned around the golfer. The system was calibrated at the start of the testing session and this calibration was used throughout. Spherical retro-reflective markers $(1.4 \mathrm{~cm})$ were adhered to the skin on anatomical regions throughout the testing session according to the adapted version of the Vicon Plug-inGait Model (Vicon Motion Systems Ltd, Oxford, UK) using double-sided tape. This model was adapted by adding an additional marker to the $4^{\text {th }}$ spinous process of the lumbar region due to the occlusion of jugular notch marker. This marker is often occluded from the camera view during the golf swing due to the head position and the left and right upper section of the arm during the backswing and downswing. Spherical markers were adhered to the golf club in four locations including the base of the grip, halfway down the club, the hosel of the club, and the club head. The four markers on the club were used to determine the top of the backswing and the initial phase of the downswing. To ensure accurate 3-D data collection, participants were asked to wear tight fitting shorts and their own golf shoes when performing all golf shots. In addition to kinematic data collection, the Voice Caddie Swing Launch Monitor SC 100 GPS 
1 (La Mirada, CA, USA) was used to calculate club head velocity, ball velocity and actual carry

2 distance of each golf shot. The Launch Monitor was previously validated in-house against the

3 Vicon Bonita Motion Analysis System; TrackmanTM III Golf Swing and Ball Flight Analysis

4 System (Brighton, MI, USA). (CHS: ICC $=0.92$, BS: ICC $=0.99)$. The Launch Monitor was

5 required to be positioned $1 \mathrm{~m}$ directly behind the golf ball and positioned towards the target

6 line of the golfer. After each golf shot, the performance variables were logged using Microsoft

$7 \quad$ Excel (Excel $2016($ v16.0)).

\section{Data Analysis}

9 For the five swings preformed pre and post practice session with the driver, the trials were 10 individually analysed and compared between the sessions. ${ }^{7}$ All Raw 3D co-ordinate data was filtered using a fourth-order low-pass Butterworth filter with a cut-off frequency of $6 \mathrm{~Hz}$. A multi-segment model used to analyse the X-Factor in the current study was developed using BodyBuilder (Oxford, UK) and used in Vicon version 2.2.2. The X-Factor was calculated by measuring the orientation of the torso in relation to the pelvis. ${ }^{17}$ The X-Factor stretch was calculated by subtracting the $\mathrm{X}$-Factor at the top backswing from the maximum $\mathrm{X}$-Factor value during the downswing. The backswing was defined as the point in which the clubhead reached its most lateral position, towards the intended target, before changing direction. The downswing is defined as the period from top of the backswing to ball contact.

\section{Statistical Analysis}

Normal distribution for all variables was assessed using the Shapiro-Wilk test. If normal distribution was not granted, a log transformation was conducted on the specific data sets. Following this, an ANCOVA was used to determine significant differences, if any, between the X-Factor and the X-Factor stretch from the five shots performed pre and post practice session. Additionally, an ANCOVA was used to determine significant differences, if any, between the club head velocity, ball velocity and actual carry distance from the five shots 
1 performed pre and post practice session. Following a $p$ value correction, the level of

2 significance was set at $p \leq 0.02$ and effect sizes were calculated using the Cohen's $d$ method. ${ }^{30}$

3 Effect sizes of less than 0.2 were considered negligible effects and between 0.2 and 0.5 were

4 considered small effects. Values between 0.5 and 0.8 were considered medium effects and

5 greater than 0.8 represented large effects. ${ }^{30}$ All calculations were performed in SPSS (version

$6 \quad 23)$ and Microsoft Excel.

\section{$7 \quad$ Results}

\section{Biomechanical Variables}

9 Significant differences were observed for the X-Factor after the completion of the golf practice session in comparison to the golf swings performed before the practice session $(p=0.00, d=$ 0.22). On average participants $X$-Factor was $52.82 \pm 5.64^{\circ}$ at the start of the golf practice compared to $54.06 \pm 5.61^{\circ}$ at the end of the golf practice session.

Significant differences were observed for the X-Factor stretch after the completion of the golf practice session in comparison to the golf swings performed before the practice session $(p=$ $0.02, d=0.25)$. On average participants $\mathrm{X}$-Factor stretch was $1.54 \pm 1.05^{\circ}$ at the start of the golf practice compared to $1.90 \pm 1.41^{\circ}$ at the end of the golf practice session.

\section{Performance Variables}

Significant changes were displayed in club head velocity after the golf practice session in comparison to the swings performed before the practice session when using the driver ( $p=$ $0.00, d=0.35)$. On average participants club head velocity was $166.79 \pm 7.81 \mathrm{~km} / \mathrm{h}$ at the start of the golf practice compared to $169.49 \pm 7.43 \mathrm{~km} / \mathrm{h}$ at the end of the golf practice session.

Significant changes were displayed in ball velocity after the golf practice session in comparison to the swings performed before the practice session when using the driver $(p=0.01, d=0.21)$. 
1 On average participants ball velocity was $237.81 \pm 14.95 \mathrm{~km} / \mathrm{h}$ at the start of the golf practice

\section{Discussion}

8 The aim of the current study was to describe the changes, if any, in the X-Factor at the top of 9 the backswing and X-Factor stretch during the initiation of the downswing during the golf compared to $240.83 \pm 13.36 \mathrm{~km} / \mathrm{h}$ at the end of the golf practice session.

Significant changes were displayed in actual carry distance after the golf practice session in comparison to the swings performed before the practice session when using the driver $(p=$ $0.00, d=0.29)$. On average participants actual carry distance was $235.44 \pm 17.77 \mathrm{~m}$ at the start of the golf practice compared to $240.14 \pm 13.88 \mathrm{~m}$ at the end of the golf practice session. swings performed pre and post golf practice session. Additionally, the current study aimed to describe the changes, if any, in club head velocity, ball velocity and actual carry distance from the golf swings performed pre and post golf practice session. A null hypothesis for all variables was adopted due to the conflicting views on the topic area.

The findings from the current study show that the X-Factor at the top of the backswing increased significantly as a result of performing multiple golf swings, therefore rejecting the initial hypothesis. It must be highlighted, however, that the effect sizes for these X-Factor comparisons were small. Although the magnitude of change was small when comparing pre and post conditions, the results demonstrate that the incorporation of multiple golf swings during a practice session could be important in producing increased coordination patterns, which can result in greater movement patterns and in turn can increase the performance of the golfer. These findings suggest that when PGA Professionals are designing golf practice and warm-up sessions for elite golfers, they should advocate that golfers hit 100 balls. As previously discussed, limited research has been conducted regarding the effect that performing multiple golf swings has on movement patterns during the golf swing. Researchers have, 
1 however, investigated the effect that various warm-up routines have on the X-Factor at the top

2 of the backswing.

3 In relation to warm-up routines, it has been previously reported that performing a golf specific

4 dynamic rotational warm-up prior to golf play does not significantly increase the X-Factor. ${ }^{15}$

5 The study conducted by Henry and colleagues has similarities to the current study. Both studies

6 have examined movements directly related to the golf swing and what effect repeating these

7 over time has on the X-Factor. The contrasting findings between the studies may be a result of

8 the multiple golf swings performed in the current study increasing ROM of the upper limbs to

9 a greater extent than performing a golf specific dynamic warm-up routine. Previous research

10 has shown that performing multiple golf swings as part of a warm-up routine significantly increases long game performance in comparison to passive static stretching. ${ }^{23}$ Gergley $^{23}$ reported club head velocity significantly increased by $6.19 \mathrm{~km} / \mathrm{h}$ when performing a warm-up protocol comprising of only golf swings, compared to performing passive static stretching. Taking these previous findings into consideration, the findings of the current study suggests that performing multiple golf shots as a warm-up method may be more beneficial than dynamic and passive static stretching. Furthermore, although the protocols have similarities in relation to increasing $\mathrm{ROM}$, there are distinct differences in the participants handicap range between the two studies, which could be a reason for the conflicting findings. The mean handicap within the study conducted by Henry and colleagues was $13.9 \pm 6.4$, whereas the handicap in the current study was $3.3 \pm 1.7$. The higher skilled golfers participating in the current study may respond differently to the intervention in comparison to the higher handicap golfers due to their increased practice hours. With regards to the X-Factor results at the top of the backswing between the two studies, Henry and colleagues reported a mean angle of $31.0 \pm 9.2^{\circ}$ prior to the dynamic stretch routine and $34.1 \pm 9.0^{\circ}$ after the warm-up. The values within the current study are considerably higher which are to be expected due to the higher skill level of golfer. 
1 It has been previously reported that there is a correlation between handicap and the X-Factor

2 at the top of the swing. As the handicap of the golfers increases, the X-Factor decreases. ${ }^{11,31}$

3 In relation to the X-Factor stretch, the current findings show significant differences between

4 the pre and post golf practice session, however, it must be highlighted that the effect sizes for

5 these comparisons were small. Although small in magnitude, the results suggest that the X-

6 Factor stretch increases following multiple golf swing. This finding is conflicting with

7 previously published literature where warm-up techniques have been analysed. Henry et al. ${ }^{15}$

8 found no significant difference in the X-Factor stretch between the pre and post golf swing

9 during a golf specific dynamic warm-up routine. These conflicting findings may be a result of

10 the previously discussed participant differences between the current study and the study conducted by Henry and colleagues. It has been noted previously that an increase in the XFactor stretch at the beginning of the downswing can increase the performance of the golfer. ${ }^{4}$

The findings from the current study support these views by displaying a significant increase in club head velocity, ball velocity and actual carry distance when the X-Factor stretch significantly increased after the completion of the practice session.

In addition to the changes in the X-Factor and X-Factor stretch, the current study displayed significant changes in club head velocity, ball velocity and actual carry distance following completion of the practice session. Similar to the X-Factor and X-Factor stretch, the effect sizes for all performance variables were small. Although the magnitude of change between pre and post practice session swings are small, these results are in agreement with the previously reported correlation between club head velocity and the X-Factor at the top of the backswing. ${ }^{4,11}$ Previous studies have reported the effect a golf practice session has on long game performance variables. Horton et al. ${ }^{7}$ reported no significant changes in ball velocity after the completion of the golf practice session. Although the authors did report an increase in ball velocity after the golf practice session. Sorbie et al. ${ }^{26}$ reported no significant changes to club 
1 head velocity, ball velocity and actual carry distance. Contrasting to the study by Horton and

2 colleagues, Sorbie et al. ${ }^{26}$ reported a decrease in club head velocity, ball velocity and actual

3 carry distance after the golf practice session was complete. The current study and the study

4 conducted by Horton et al. $^{7}$ tested low handicap golfers and professional respectively. In

5 contrast, Sorbie et al. ${ }^{26}$ examined higher handicap golfers $(15.2 \pm 5.7)$. These contrasting

6 findings may be a result of the higher handicap golfers conducting less practice time compared

7 to skilled golfers and as a result display signs of muscular fatigue during the repetitive golf

8 shots. $^{26}$

9 To the researchers knowledge, only Horton et al. ${ }^{7}$ and Sorbie et al. ${ }^{26}$ have investigated the effect

10 that the golf practice session has on injury prevention and specific performance variables associated with the long game. In terms of injury, Horton et al. ${ }^{7}$ found that performing practice sessions is unlikely to increase injury in the trunk area during the golf swing. Similarly, Sorbie et al. $^{26}$ reported no change in erector spinae muscle activation during the golf swing when performing a golf practice session. The current study adds to this topic by displaying increases in the X-Factor, X-Factor stretch and long game performance variables during the completion of a golf practice session within low-handicap golfers. These findings can help PGA Professionals, who teach highly skilled golfers, to improve movement patterns in relation to the X-Factor and X-Factor stretch and, in turn, improve the long game performance of the golfer. The findings of the current study can also help PGA Professionals to design golf practice sessions and warm-up sessions that can improve the long game of the golfer. Specifically, as a result of the findings from the current study, PGA Professionals can promote that golfers should hit 100 balls during a practice session or warm-up session in order to optimize movement patters and long game performance. Implementing this prior to golf play or competition may enable highly skilled golfers to be at an optimal level at the beginning of the golf round. 
1 However, future studies should examine the performance of the golf round following the

2 practice session in the current study.

3 Following the reported increase in the X-Factor at the top of the backswing, and marginal

4 increases in club head velocity, ball velocity and actual carry distance, further investigation is

5 required to ascertain the point where the X-Factor and performance variables are at their

6 optimal throughout the golf practice session. This could be an important finding for

7 practitioners when designing golf practice sessions and golf specific warm-up sessions for

8 professional and amateur golfers. It is important for future research to be conducted using

9 participants with a higher handicap and also female golfers in order to increase the

10 understanding of the topic area. In addition to this, it is also important for future search to

11 investigate additional movement patterns throughout the golf swing that are associated with

12 increased performance.

13 It is important to acknowledge the limitations within the current study. All golf shots performed

14 by the participants were hit with the identical clubs and shaft lengths. Although participants were given time to familiarise themselves with the two clubs and shaft lengths used within the current study, it has been previously suggested swing kinematics are influenced by a golfer hitting a non-familiar club and shafts. ${ }^{32}$ However, standardising the golf clubs throughout the study removes the effect of the golf club from the results.

\section{Conclusion}

The results of the current study indicate that performing multiple golf swings can increase the $\mathrm{X}$-Factor at the top of the backswing and can increase the X-Factor stretch during the initiation of the downswing. Furthermore, the results from the current study indicate that performing multiple golf swings increases long game performance variables. These results demonstrate that performing multiple golf swings could be important in order to produce increased 
coordination patterns, which can result in greater movement patterns of the golfer and therefore increase long game performance. These findings can be important for PGA Professionals in terms of better understanding of the efficacy of golf practice and warm-up sessions in relation to golf performance.

\section{Funding}

The author(s) received no financial support for the research, authorship, and/or publication of this article.

\section{Disclosure statement}

No potential conflict of interest was reported by the authors.

\section{References}

1. Doan BK, Newton RU, Kwon Y-H, et al. Effects of physical conditioning on intercollegiate golfer performance. J Strength Cond Res 2006; 20: 62-72.

2. Fradkin A, Sherman C, Finch C. How well does club head speed correlate with golf handicaps? J Sci Med Sport 2004; 7: 465-472.

3. Thompson C, Blackwell J. Functional training improves club head speed and functional fitness in older golfers. J Strength Cond Res 2007; 21: 131-137.

4. Lephart S, Smoliga J, Myers J, et al. An eight-week golf-stretching exercise program improves physical characteristics, swing mechanics, and golf performance in recreational golfers. J Strength Cond Res 2007; 21: 860-869.

5. Fletcher IM, Hartwell M. Effect of an 8-week combined weights and plyometrics training program on golf drive performance. J strength Cond Res 2004; 18: 59-62.

6. Hetu F, Christie C, Faigenbaum A. Effects of conditioning on physical fitness and club head speed in mature golfers . Percept Mot Skills 1998; 86: 811-815.

7. Horton J, Lindsay D, Macintosh B. Abdominal muscle activation of elite male golfers with chronic low back pain. Med Sci Sport Exerc 2001; 1647-1654.

8. Joyce C. An examination of the correlation amongst trunk flexibility, $\mathrm{x}$-factor and clubhead speed in skilled golfers. J Sports Sci 2016; 1-7.

9. An J, Wulf G, Kim S. Increased carry distance and X-Factor stretch in golf through an external focus of attention. J Mot Learn Dev 2013; 1: 2-11.

10. Tinmark F, Hellström J, Halvorsen K, et al. Elite golfers' kinematic sequence in fullswing and partial-swing shots. Sport Biomech 2010; 9: 236-44.

11. Myers J, Lephart S, Tsai Y-S, et al. The role of upper torso and pelvis rotation in 
driving performance during the golf swing. J Sports Sci 2008; 26: 181-188.

12. Nesbit SM. A three dimensional kinematic and kinetic study of the golf swing. $J$ Sports Sci Med 2005; 4: 499-519.

13. Healy A, Moran KA, Dickson J, et al. Analysis of the 5 iron golf swing when hitting for maximum distance. J Sports Sci 2011; 29: 1079-88.

14. Hume PA, Keogh J, Reid D. The role of biomechanics in maximising distance and accuracy of golf shots. Sport Med 2005; 35: 429-449.

15. Henry E, Berglund K, Millar L, et al. Immediate effects of a dynamic rotation-specific warm-up on $\mathrm{x}$-factor and $\mathrm{x}$-factor stretch in the amateur golfer. Int J Sports Phys Ther 2015; 10: 998-1007.

16. Mclean J, Andrisani J. The X-factor swing: And other secrets to power and distance. New York: Harper Collins, 1996.

17. Brown SJ, Selbie WS, Wallace ES. The X-Factor: An evaluation of common methods used to analyse major inter-segment kinematics during the golf swing. J Sports Sci 2013; 31: 1156-63.

18. Chettle DK, Neal RJ. Optimising performance in golf. In: Ltd. AAP (ed) Strength and conditioning for golf. Brisbane, 2001, pp. 207-223.

19. Keogh JW, Marnewick MC, Maulder PS, et al. Are anthropometric, flexibility, muscular strength, and endurance variables related to clubhead velocity in low- and high-handicap golfers? J Strength Cond Res 2009; 23: 1841-1850.

20. Lindsay DM, Horton JF. Trunk rotation strength and endurance in healthy normals and elite golfers with and without lower back pain. North Am J Sport Phys Ther 2006; 1: 80-89.

21. Radford JA, Burns J, Buchbinder R, et al. Does stretching increase ankle dorsiflexion range of motion? A systematic review. Br J Sports Med 2006; 40: 870-875.

22. Stewart IB, Sleivert GG. The effect of warm-up intensity on range of motion and anaerobic performance. J Orthop Sport Phys Ther 1998; 27: 154-161.

23. Gergley J. Acute effects of passive stratic stretching during warm-up on driver clubhead speed, distance, accuracy, and consistent ball contact in young male competitive golfers. J strength Cond Res 2009; 23: 863-867.

24. Tilley NR, Macfarlane A, Tilley N. Effects of different warm-up programs on golf performance in elite male golfers. Int J fo Sport Phys Ther 2012; 7: 388-395.

25. Higdon NR, Finch WH, Leib D, et al. Effects of fatigue on golf performance. Sport Biomech 2012; 11: 190-196.

26. Sorbie GG, Baker JS, Gu Y, et al. Comparison of thoracic and lumbar erector spinae muscle activation before and after a golf practice session. J Appl Biomech 2017; 33: 288-293.

27. Okuda I, Gribble P, Armstrong C. Trunk rotation and weight transfer patterns between skilled and low skilled golfers. J Sport Sci Med 2010; 9: 127-133.

28. Sorbie GG, Grace FM, Gu Y, et al. Electromyographic analyses of the erector spinae 
muscles during golf swings using four different clubs. J Sports Sci 2018; 36: 717-723.

29. Silva L, Vaz JR, Castro MA, et al. Recurrence quantification analysis and support vector machines for golf handicap and low back pain EMG classification. $J$ Electromyogr Kinesiol 2015; 25: 637-647.

5 30. Cohen J. A Power Primer. Psychol Bullitin 1992; 112: 155-159.

6 31. Zheng N, Barrentine S, Fleisig G, et al. Kinematic analysis of swing in pro and

32. Joyce C, Burnett A, Cochrane J, et al. A preliminary investigation of trunk and wrist kinematics when using drivers with different shaft properties. Sport Biomech 2016; 15: 61-75. 